\title{
Posterior Probabilistic Clustering using NMF
}

\author{
Chris Ding \\ CSE Department \\ University of Texas at Arlington \\ chqding@uta.edu \\ Dijun Luo \\ CSE Department \\ University of Texas at Arlington \\ dijun.luo@gmail.com
}

\author{
Tao Li \\ School of Computer Science \\ Florida International. University \\ taoli@cs.fiu.edu \\ Wei Peng \\ School of Computer Science \\ Florida International University \\ wpeng002@cs.fiu.edu
}

\begin{abstract}
We introduce the posterior probabilistic clustering (PPC), which provides a rigorous posterior probability interpretation for Nonnegative Matrix Factorization (NMF) and removes the uncertainty in clustering assignment. Furthermore, PPC is closely related to probabilistic latent semantic indexing (PLSI).
\end{abstract}

\section{Categories and Subject Descriptors}

H.3.3 [Information Search and Retrieval]: Clustering; I.2 [Artificial Intelligence]: Learning

\section{General Terms}

Algorithms, Experimentation, Measurement, Performance, Theory

\section{Keywords}

Sparse, Posterior Probabilistic Clustering, NMF

\section{INTRODUCTION}

Non-negative Matrix Factorization (NMF) [4] has been successfully applied to document clustering recently $[5,1]$. However, in the standard NMF clustering, cluster assignment is rather ad hoc. In addition, matrix factors lack clear interpretations.

In this work, we introduce the posterior probabilistic clustering (PPC), which has 3 benefits: (1) It provides a rigorous posterior probability interpretation for both matrix factors $F, G$ in the factorization of input $X: X \simeq F G^{T}$. (2) It removes the uncertainty in clustering assignment. (3) Furthermore, when we perform simultaneous word and document clustering, the new model has a very close relation to probabilistic latent semantic indexing (PLSI) [3]: in PLSI, $F, G$ are class conditional probabilities; in PPC, $F, G$ are class posterior probabilities.

Copyright is held by the author/owner(s). SIGIR'08, July 20-24, 2008, Singapore. ACM 978-1-60558-164-4/08/07.

\section{STANDARD NMF CLUSTERING}

Suppose we have $n$ documents and $m$ words (terms). Let $X=$ $\left(X_{i j}\right)$ be the word-to-document matrix: $X_{i j}=X\left(w_{i}, d_{j}\right)$ is the frequency of word $w_{i}$ in document $d_{j}$. Standard NMF optimization is

$$
\min _{F \geq 0, G \geq 0}\left\|X-F G^{T}\right\|^{2},
$$

where $X$ has size $m \times n, F$ has size $m \times K, G$ has size $n \times K$. Once the solution $\left(F^{*}, G^{*}\right)$ is computed, standard approach is to assign $d_{j}$ to the cluster $C_{k}$ where

$$
k=\arg \max \left(G_{j 1}^{*}, \cdots, G_{j K}^{*}\right),
$$

i.e., the largest element of $j$-th row of $G$.

There is a fundamental problem with this approach. First, the solution to NMF is not unique. For an arbitrary positive diagonal matrix $D=\operatorname{diag}\left(d_{1}, \cdots, d_{k}\right)$, we have

$$
F^{*} G^{* T}=\left(F^{*} D^{-1}\right)\left(G^{*} D\right)^{T}
$$

i.e., $\left(F^{*} D^{-1}, G^{*} D\right)$ is also an optimal solution. Thus the cluster assignment is modified to

$$
k=\arg \max \left(G_{j 1}^{*} d_{1}, \cdots, G_{j k}^{*} d_{K}\right) .
$$

A different choice of $D$ leads to different cluster assignment. An ad hoc solution is to choose $D$ such that columns of $F$ have unit length in $L_{2}$ norm.

\section{POSTERIOR PROBABILITY}

In this work, we present a principled way to resolve this problem. This is based on posterior probability interpretation of $G$. In fact, we can see from Eq.(3) that (roughly speaking)

$$
\left(G_{j 1}^{*} d_{1}, \cdots, G_{j k}^{*} d_{K}\right),
$$

is the posterior probability that $d_{j}$ belongs to different clusters. Thus we wish to choose $D$ such that

$$
G_{j 1}^{*} d_{1}+\cdots+G_{j k}^{*} d_{K}=1, \quad j=1, \cdots, n
$$

This requirement has no solution, because there are $n$ constraints and $K$ variables, but $K$ is much less $n$. Therefore, in standard NMF, there is no way to enforce posterior probability normalization. 


\section{POSTERIOR PROBABILISTIC CLUSTER- ING}

In our approach, we enforce the posterior probability normalization directly. The posterior probabilistic clustering is to optimize

$$
\min _{F \geq 0, G \geq 0}\left\|X-F G^{T}\right\|^{2}, \text { s.t. } \sum_{k=1}^{K} G_{j k}=1,
$$

Using Lagrangian multipliers to enforce the constraints, we derive the following updating rules to solve this problem

$$
\begin{gathered}
G_{i k} \leftarrow G_{i k} \frac{\left(X^{T} F\right)_{i k}+\left(G F^{T} F G^{T}\right)_{i i}}{\left(G F^{T} F\right)_{i k}+\left(X^{T} F G^{T}\right)_{i i}} \\
F_{i k} \leftarrow F_{i k} \frac{\left(X G^{T}\right)_{i k}}{\left(F G^{T} G\right)_{i k}}
\end{gathered}
$$

The correctness and convergence can be proved rigorously. In the updating process, the constraints should be enforced periodically.

\section{SIMULTANEOUS WORD AND DOCUMENT CLUSTERING (SPPC)}

We generalize PPC to simultaneous word and document clustering. We use $F$ as the posterior probability for word clustering, and the posterior probability normalization is $\sum_{k=1}^{K} F_{i k}=1$. The simultaneous PPC (SPPC) problem becomes

$$
\min _{F \geq 0, S, G \geq 0}\left\|X-F S G^{T}\right\|^{2} \text {, s.t. } \sum_{k=1}^{K} F_{i k}=1, \sum_{k=1}^{K} G_{j k}=1,
$$

We derived the updating algorithm as follows. Let $\widetilde{F}=F S, \widetilde{G}=$ $G S^{T}$, the updating algorithm is

$$
\begin{gathered}
G_{i k} \leftarrow G_{i k} \frac{\left(X^{T} \widetilde{F}\right)_{i k}+\left(G \widetilde{F}^{T} \widetilde{F} G^{T}\right)_{i i}}{\left(G \widetilde{F}^{T} \widetilde{F}\right)_{i k}+\left(X^{T} \widetilde{F} G^{T}\right)_{i i}} \\
F_{i k} \leftarrow F_{i k} \frac{\left(X \widetilde{G}^{T}\right)_{i k}+\left(F \widetilde{G}^{T} \widetilde{G} F^{T}\right)_{i i}}{\left(F \widetilde{G}^{T} \widetilde{G}\right)_{i k}+\left(X \widetilde{G} F^{T}\right)_{i i}} \\
S_{k k^{\prime}} \leftarrow S_{k k^{\prime}} \frac{\left(F^{T} X G\right)_{k k^{\prime}}}{\left(F^{T} F S G^{T} G\right)_{k k^{\prime}}}
\end{gathered}
$$

We initialize $F, G$ to the K-means clustering results on words $F_{0}$ and on documents $G_{0}$. where $F_{0}, G_{0}$ are cluster indicators. We set $F=F_{0}+0.2$ and $G=G_{0}+0.2$.

\subsection{Relation to PLSI}

In PLSI we view the word-document matrix $X$ as the joint probability of word and documents. [We re-scale the term frequency $X_{i j}$ by $X_{i j} \leftarrow X_{i j} / T_{w}$, where $T_{w}=\sum_{i j} X_{i j}$. With this, $\sum_{i j} X_{i j}=$ 1. ] The joint occurrence probability is $p\left(w_{i}, d_{j}\right)=X_{i j}$. PLSI decompose it as product of class-conditional probabilities:

$$
X_{i j} \approx \sum_{k} P\left(\text { word }_{i} \mid \text { class }_{k}\right) P\left(\text { class }_{k}\right) P\left(\text { doc }_{j} \mid \text { class }_{k}\right) .
$$

Let $F_{i k}=P\left(\right.$ word $_{i} \mid$ class $\left._{k}\right), S_{k k}=P\left(\right.$ class $\left._{k}\right), G_{j k}=P\left(\right.$ doc $_{j} \mid$ class $\left._{k}\right)$ PLSI optimization problem is:

$$
\min _{F \geq 0, S, G \geq 0} \operatorname{Dist}\left(X, F S G^{T}\right) \text {, s.t. } \sum_{i=1}^{m} F_{i k}=1, \sum_{j=1}^{n} G_{i k}=1,
$$

Therefore, our SPPC is quite similar to PLSI, except SPPC has a different normalization $\sum_{k=1}^{K} G_{j k}=1, \sum_{k=1}^{K} F_{i k}=1$. In other words, SPPC treats $G_{j k}, F_{i k}$ as posterior probabilities; PLSI treats $G_{j k}, F_{i k}$ as class-conditional probabilities.

Note that in PLSI, the sum of probabilities of a document belong to different classes, $\sum_{k=1}^{K} G_{j k}=P\left(\right.$ doc $_{j} \mid$ class $\left._{k}\right) \neq 1$. Intuitively for clustering, we would like the total probability adds up to 1 . This deficiency is removed in SPPC.

\subsection{An Illustrative example}

We give a simple example to illustrate the PPC and SPPC results. The data matrix is given bellow. From inspection, first 3 columns belong to one cluster and the last 4 columns belong to another. For rows, first 3 rows belong to one cluster and the last 2 row belong to another. The resulting $F, G$ recover the clustering correctly.

$$
\begin{aligned}
X & =\left(\begin{array}{lllllll}
0.185 & 0.326 & 0.761 & 2.799 & 2.375 & 2.970 & 2.585 \\
0.508 & 0.380 & 0.884 & 2.134 & 2.374 & 2.342 & 2.524 \\
0.452 & 0.887 & 0.457 & 2.065 & 2.484 & 2.253 & 2.163 \\
1.486 & 1.843 & 1.858 & 0.566 & 0.103 & 0.417 & 0.269 \\
1.496 & 1.806 & 1.610 & 0.612 & 0.158 & 0.560 & 0.784
\end{array}\right) \\
F_{\mathrm{PPC}}^{T} & =\left(\begin{array}{lllllll}
0.068 & 0.059 & 0.056 & 0.000 & 0.005 \\
0.007 & 0.011 & 0.012 & 0.037 & 0.035
\end{array}\right) \\
G_{\mathrm{PPC}}^{T} & =\left(\begin{array}{lllllll}
0.003 & 0.001 & 0.077 & 0.763 & 0.835 & 0.836 & 0.795 \\
0.997 & 0.999 & 0.923 & 0.237 & 0.165 & 0.164 & 0.205
\end{array}\right) \\
F_{\mathrm{SPPC}}^{T} & =\left(\begin{array}{lllllll}
0.961 & 0.830 & 0.792 & 0.019 & 0.091 \\
0.039 & 0.170 & 0.208 & 0.981 & 0.909
\end{array}\right) \\
G_{\mathrm{SPPC}}^{T} & =\left(\begin{array}{lllllll}
0.033 & 0.031 & 0.113 & 0.846 & 0.922 & 0.924 & 0.880 \\
0.967 & 0.969 & 0.887 & 0.154 & 0.078 & 0.076 & 0.120
\end{array}\right)
\end{aligned}
$$

\section{EXPERIMENTS}

We compare the clustering performance of each method on 5 real-life datasets. More details of these datasets can be found in [2]. We use accuracy as the performance measure. The experimental results are shown in Table 1. We see that SPPC performs slightly better than NMF and PLSI.

\begin{tabular}{l|r|r|r|r}
\hline \hline Datasets & K-Means & NMF & PLSI & SPPC \\
\hline CSTR & 0.4256 & 0.5713 & 0.587 & 0.5945 \\
WebKB4 & 0.3888 & 0.4418 & 0.503 & 0.4411 \\
Log & 0.6876 & 0.7805 & 0.778 & 0.7915 \\
Reuters & 0.4448 & 0.4947 & 0.4870 & 0.5648 \\
WebAce & 0.4001 & 0.4761 & 0.4890 & 0.4953 \\
\hline \hline
\end{tabular}

Table 1: Clustering Results. Shown are the accuracy results for different methods.

\section{REFERENCES}

[1] C. Ding, X. He, and H.D. Simon. On the equivalence of nonnegative matrix factorization and spectral clustering. Proc. SIAM Data Mining Conf, 2005.

[2] C. Ding, T. Li, W. Peng, and H. Park. Orthogonal nonnegative matrix t-factorizations for clustering. In KDD'06, pages 126-135, 2006.

[3] T. Hofmann. Probabilistic latent semantic analysis. In ACM SIGIR-99, pages 289-296, 1999.

[4] D.D. Lee and H. S. Seung. Algorithms for non-negative matrix factorization. In Advances in Neural Information Processing Systems, volume 13 .

[5] W. Xu, X. Liu, and Y. Gong. Document clustering based on non-negative matrix factorization. In Proc. ACM conf. Research and development in IR(SIGIR), pages 267-273, Toronto, Canada, 2003. 